九州大学学術情報リポジトリ

Kyushu University Institutional Repository

\title{
ON A CLASS OF NONLINEAR PROGRAMMING IN A BANACH SPACE
}

Tagawa, Shojiro

International Institute for Advanced Study of Social Information Science

https://doi.org/10.5109/13086

出版情報: 統計数理研究. 16 (1/2)，pp. 95-114，1974-03. Research Association of Statistical Sciences

バージョン：

権利関係 : 


\title{
ON A CLASS OF NONLINEAR PROGRAMMING IN A BANACH SPACE
}

\author{
By
}

\author{
Shôjirô TAGAwA*
}

(Received October 15, 1973)

\section{Introduction.}

This paper is devoted to present the optimality conditions for the minimum in a Banach space. More specifically, we shall be ultimately concerned with the following problem:

$$
\begin{array}{ll}
\text { minimize } & f(x) \\
\text { subject to } & x \in A \\
& g(x) \in B,
\end{array}
$$

where the objective function $f$ is a real valued function on a Banach space $X, A$ is an arbitrary subset of $X, g$ is a mapping of $X$ into a Banach space $Y$, and $B$ is a closed convex cone with vertex at the origin in $Y$, which has a non-empty interior.

In order to discuss the above problem we start in Section 2 with the preparation of some fundamental notions which are indispensable for our approach in this paper. It is noted that the local closed convex cone of $A$ at a point $\bar{x}$ and the pseudoconvex function $f$ are crucial in our approach. In Section 3, nonlinear programming in a Banach space is discussed. Theorems 3.1 and 3.2 are concerned with the problem : minimize $\{f(x) \mid x \in A\}$, where $A$ is a set in a Banach space $X$, and $f$ is a real valued function defined over $X$. The necessary conditions for the problem $(\mathrm{P})$ are obtained in Theorem 3.3 under the assumptions that $f$ and $g$ are both differentiable in the sense of Neustadt [6], and that $A$ is locally convex at $\bar{x}$ or is locally relatively convex with respect to $H=g^{-1}(B)$ at $\bar{x}$. Theorem 3.4 presents the sufficient conditions for the problem (P) when our objective function is pseudoconvex and the constraint set $A \cap H$ is taken as pseudocone. The saddle value problem ( $\mathrm{P}^{\prime}$ ) associated with the original problem $(\mathrm{P})$ is discussed in Section 3. The applications of Theorems 3.3 and 3.4 yield us Theorems 3.6 and 3.7, which show the interrelation between these two problems.

In Section 4, we apply the main results given in Theorems 3.3 and 3.4 to the ordinary convex programming in a Banach space since every continuous convex function is differentiable in the sense of Neustadt. Section 5 is devoted to discuss the implication of our assumptions given in Theorem 3.3 in comparison with (i) those of Varaiya [8] and (ii) those of Neustadt [5] or Nagahisa \& Sakawa [4].

\footnotetext{
* International Institute for Advanced Study of Social Information Science.
} 


\section{Notations and preliminaries.}

Let $X$ and $Y$ be real linear topological spaces, $A$ a non-empty subset of $X$ and $\bar{x} \in A$. We define the cone generated by $A$ as follows:

$$
\text { cone }(A)=\{\lambda a \mid \lambda>0, a \in A\} \text {. }
$$

Definition 2.1. (Varaiya [8]). By the closed cone of $A$ at $\bar{x}$ we mean the intersection of all closed cones* containing the set

$$
A-\bar{x}=\{a-\bar{x} \mid a \in A\} .
$$

We denote this set by $\mathrm{C}(A, \bar{x})$.

DEFinition 2.2. By the closed convex cone of $A$ at $\bar{x}$ we mean the intersection of all closed convex cones containing the set $A-\bar{x}$. We denote this set by $\operatorname{CC}(A, \bar{x})$.

Definition 2.3. (Varaiya [8]). By the local closed cone of $A$ at $\bar{x}$ we mean the set

$$
\mathrm{LC}(A, \bar{x})=\bigcap_{N \in \Re(\bar{x})} \mathrm{C}(A \cap N, \bar{x}),
$$

where $\mathfrak{R}(\bar{x})$ is the class of all neighborhoods of $\bar{x}$.

Definition 2.4. By the local closed convex cone of $A$ at $\bar{x}$ we mean the set

$$
\mathrm{P}(A, \bar{x})=\bigcap_{N \in \mathscr{R}(\bar{x})} \mathrm{CC}(A \cap N, \bar{x}) .
$$

It is easily seen that $\mathrm{P}(A, \bar{x})$ is a closed convex cone and that

$$
\mathrm{LC}(A, \bar{x}) \subset \mathrm{P}(A, \bar{x}) \text {. }
$$

Now, we can state the following lemmas.

Lemma 2.1. (Varaiya [8]). Let $X$ be a Banach space and $\bar{x} \in A$. The vector $z \in \operatorname{LC}(A, \bar{x})$ if and only if there exists a sequence of vectors $\left\{x_{n}\right\} \subset A$ and a sequence of positive numbers $\left\{\lambda_{n}\right\}$ such that

$$
\begin{aligned}
& x_{n} \longrightarrow \bar{x} \text { as } n \longrightarrow \infty \text {, } \\
& \lambda_{n}\left(x_{n}-\bar{x}\right) \longrightarrow z \text { as } n \longrightarrow \infty \text {. }
\end{aligned}
$$

Lemma 2.2. Let $X$ be a Banach space and $\bar{x} \in A$. The vector $z \in \mathrm{P}(A, \bar{x})$ if and only if there exist a sequence of positive numbers $\left\{\lambda_{n}\right\}$, a sequence of positive integers $\left\{m_{n}\right\}$, a sequence of vectors $\left(\alpha_{n 1}, \cdots, \alpha_{n m_{n}}\right) \in P^{m_{n}}$, and a sequence of vectors $\left\{y_{n i}\right\}_{i=1, \cdots, m_{n}}$ $\subset A$ such that

$$
\begin{aligned}
y_{n i} \stackrel{n \longrightarrow \infty}{\longrightarrow} \bar{x} \text { uniformly in } i, \\
\lambda_{n}\left(\sum_{i=1}^{m_{n}} \alpha_{n i} y_{n i}-\bar{x}\right) \stackrel{ }{\longrightarrow} z,
\end{aligned}
$$

where $P^{l}$ is the subset of $R^{l}$ (l-Eucledian space) defined by

$$
P^{l}=\left\{\left(\alpha_{1}, \cdots, \alpha_{l}\right) \in R^{l} \mid \alpha_{i} \geqq 0, i=1, \cdots, l, \sum_{i=1}^{l} \alpha_{i}=1\right\} .
$$

* A set $A$ in a linear space will be called a cone with vertex at $p$ if $p+\lambda(q-p) \in A$ whenever $q \in A$ and $\lambda \geqq 0$. If a set is called a cone, it means a cone with vertex at the origin. 
PROOF. First of all, it is easy to verify that

$$
\mathrm{CC}(A, \bar{x})=\mathrm{C}(\operatorname{co}(A), \bar{x})=\overline{\operatorname{cone}(\operatorname{co}(A)-\bar{x})} .^{*}
$$

We shall denote by $N_{n}(z)$ the open spheres centered at $z$ with radious $1 / n, n=1,2, \cdots$. If $z \in \mathrm{P}(A, \bar{x})$, then

$$
\begin{aligned}
z \in \mathrm{P}(A, \bar{x}) & =\bigcap_{N=\Re(\bar{x})} \operatorname{CC}(A \cap N, \bar{x}) \\
& =\bigcap_{n} \operatorname{CC}\left(A \cap N_{n}(\bar{x}), \bar{x}\right) \\
& =\bigcap_{n} \overline{\operatorname{cone}\left(\operatorname{co}\left(A \cap N_{n}(\bar{x})\right)-\bar{x}\right)},
\end{aligned}
$$

and hence

$$
N_{n}(z) \cap \text { cone }\left(\operatorname{co}\left(A \cap N_{n}(\bar{x})\right)-\bar{x}\right) \neq \emptyset \quad \text { for all } n=1,2, \cdots .
$$

Therefore, there exist a sequence of positive numbers $\left\{\lambda_{n}\right\}$ and a seqnence of vectors $x_{n} \in \operatorname{co}\left(A \cap N_{n}(\bar{x})\right) \subset N_{n}(\bar{x})$ such that

$$
\lambda_{n}\left(x_{n}-\bar{x}\right) \in N_{n}(z) \quad \text { for } n=1,2, \cdots .
$$

Furthermore, there are a sequence of positive integers $\left\{m_{n}\right\}$, a sequence of vectors $\left(\alpha_{n 1}, \cdots, \alpha_{n m_{n}}\right) \in P^{m_{n}}$, and a sequence of vectors $\left\{y_{n i}\right\}_{i=1, \cdots, m_{n}} \subset A \cap N_{n}(\bar{x})$, such that

$$
x_{n}=\sum_{i=1}^{m_{n}} \alpha_{n i} y_{n i} \quad \text { for } \quad n=1,2, \cdots .
$$

It is then valid that

$$
\begin{aligned}
y_{n i} \stackrel{n \longrightarrow \infty}{\longrightarrow} \bar{x} \quad \text { uniformly in } i, \\
\lambda_{n}\left(\sum_{i=1}^{m_{n}} \alpha_{n i} y_{n i}-\bar{x}\right) \stackrel{ }{\longrightarrow \longrightarrow \infty} z .
\end{aligned}
$$

Conversely, let $z$ be the vector satisfying the conditions in the lemma. Since the sequence $\left\{y_{n i}\right\}_{i=1, \cdots, m_{n}} \subset A$ converges to $\bar{x}$ uniformly in $i$, for each neighborhood $N$ of $\bar{x}$ there is a positive integer $n_{0}$ such that

$$
y_{n i} \in N, \quad i=1, \cdots, m_{n} \quad \text { for all } n \geqq n_{0} .
$$

It is then true that

$$
\sum_{i=1}^{m_{n}} \alpha_{n i} y_{n i} \in \operatorname{co}(A \cap N) \quad \text { for all } n \geqq n_{0} .
$$

Hence, we have

$$
\lambda_{n}\left(\sum_{i=1}^{m_{n}} \alpha_{n i} y_{n i}-\bar{x}\right) \in \operatorname{cone}(\operatorname{co}(A \cap N)-\bar{x}) .
$$

Letting $n$ tend to $\infty$, we obtain

$$
z \in \overline{\operatorname{cone}(\operatorname{co}(A \cap N)-\bar{x})}=\mathrm{CC}(A \cap N, \bar{x}) .
$$

Since $N$ is arbitrary, we conclude that

$$
z \in \bigcap_{N=\Re(\bar{x})} \mathrm{CC}(A \cap N, \bar{x})=\mathrm{P}(A, \bar{x}) .
$$

* For any subset $A$ of a linear topological space, $\bar{A}$ denotes the closure of $A, \operatorname{co}(A)$ denotes the convex hull of $A$. 
This completes the proof of Lemma 2.2.

Definition 2.5. Let $f$ be a real valued function defined on a subset $A$ of $X$. We denote by $\mathrm{G}(f, A)$ the graph of $f$ on $A$ :

$$
\mathrm{G}(f, A)=\{(x, f(x)) \mid x \in A\} .
$$

By the local closed cone of the graph of $f$ on $A$ at $\bar{x}(\in A)$ we mean the set

$$
\mathrm{LG}(f, A, \bar{x})=\mathrm{LC}(\mathrm{G}(f, A),(\bar{x}, f(\bar{x}))) .
$$

Definition 2.6. For a subset $A$ of $X$, we define

$$
A^{-}=\left\{a^{*} \in X^{*} \mid a^{*}(a) \leqq 0 \text { for all } a \in A\right\},
$$

where $X^{*}$ is a conjugate space of $X$ which is given the $X$-topology (see Dunford and Schwartz [2]).

Note that the set $A^{-}$is a closed convex cone with vertex at the origin in $X^{*}$.

LEMma 2.3. (Guignard [3]). If $A$ is a cone in a locally convex linear topological space, then

$$
A^{-}=(\overline{\operatorname{co}(A)})^{-} .
$$

Definition 2.7. Let be given a set $A$ in a linear topological space $X$ which contains a vector $\bar{x}$. The set $A$ is called a pseudocone with vertex at $\bar{x}$ if

$$
x-\bar{x} \in \operatorname{LC}(A, \bar{x}) \quad \text { for all } x \in A \text {. }
$$

It is easily verified that if $A$ is a cone with vertex at $\bar{x}$, or is convex, then $A$ is a pseudocone with vertex at $\bar{x}$.

\section{Nonlinear programming in a Banach space.}

In this section, we shall state the main results in this paper.

Let $f$ be a real valued continuous function over a Banach space $X$, and let $A$ be an arbitrary subset of $x$. Then the following theorem holds.

THEOREM 3.1. Let $W^{+}=X \times\left\{r \in R^{1} \mid r \geqq 0\right\}$. If $\bar{x} \in A$ is an optimal solution to the problem:

then the condition

$$
\text { minimize }\{f(x) \mid x \in A\} \text {, }
$$

$$
\mathrm{LG}(f, A, \bar{x}) \subset W^{+}
$$

holds.

Proof. Let $(x, r)$ be an arbitrary element of $\operatorname{LG}(f, A, \bar{x})$. It then follows from Lemma 2.1 that there exist a sequence of positive numbers $\left\{\lambda_{n}\right\}$ and a sequence of vectors $\left(x_{n}, f\left(x_{n}\right)\right) \in \mathrm{G}(f, A)$ such that

$$
\begin{array}{r}
\left(x_{n}, f\left(x_{n}\right)\right) \stackrel{ }{n} \longrightarrow \infty \\
\lambda_{n}\left(\left(x_{n}, f\left(x_{n}\right)\right)-(\bar{x}, f(\bar{x}))\right) \stackrel{ }{ } \longrightarrow \infty(x, r) .
\end{array}
$$

Since $\bar{x} \in A$ is a minimal point of $f$ over $A$, we have 
Hence, it is clear that

$$
f\left(x_{n}\right) \geqq f(\bar{x}) \quad \text { for } \quad n=1,2, \cdots .
$$

$$
\lim _{n \rightarrow \infty} \lambda_{n}\left(f\left(x_{n}\right)-f(\bar{x})\right)=r \geqq 0 .
$$

This completes the proof of Theorem 3.1.

DEFinition 3.1. (Neustadt [6]). The function $f$ from an open set $D$ in a linear topological space $X$ into a linear topological space $Y$ is called differentiable at $\bar{x} \in D$ in the sence of Neustadt if to every $x \in X$ there corresponds a vector $f_{\bar{x}}(x) \in Y$ such that

$$
\frac{f(\bar{x}+\varepsilon y)-f(\bar{x})}{\varepsilon} \stackrel{ }{\begin{array}{l}
\varepsilon \\
y
\end{array}} \longrightarrow f_{\bar{x}}(x) .
$$

Note that the differential $f_{\bar{x}}$ is necessarily positively homogeneous, i. e.,

$$
f_{\bar{x}}(\alpha x)=\alpha f_{\bar{x}}(x) \quad \text { for all } \alpha \geqq 0 \text { and all } x \in X .
$$

LEMMA 3.1. Let $f$ be defined as above. If $f$ is differentiable at $\bar{x}$ in the sence of Neustadt, then $f$ is continuous at $\bar{x}$.

PROOF. Let $N$ be an arbitrary balanced neighborhood (see [1]) of the origin in $Y$. Then, there are a positive number $\varepsilon, 0<\varepsilon<1$, and a neighborhood $U$ of the origin in $X$ such that

$$
\frac{f(\bar{x}+\varepsilon y)-f(\bar{x})}{\varepsilon} \in f_{\bar{x}}(0)+N=N \quad \text { for all } y \in U,
$$

and hence we have

$$
f(\bar{x}+x)-f(\bar{x}) \in \varepsilon N \subset N \quad \text { for all } x \in \varepsilon U .
$$

Since $\varepsilon U$ is also a neighborhood of the origin in $X$, this implies the continuity of $f$ at $\bar{x}$.

Proposition 3.1. If $f$ is differentiable at $\bar{x} \in A$ in the sense of Neustadt, then it is true that

$$
\mathrm{LG}(f, A, \bar{x})=\left\{\left(x, f_{\bar{x}}(x)\right) \mid x \in \mathrm{LC}(A, \bar{x})\right\} .
$$

Proof. Let $C=\left\{\left(x, f_{\bar{x}}(x)\right) \mid x \in \mathrm{LC}(A, \bar{x})\right\}$. For each element $(x, r) \in \operatorname{LG}(f, A, \bar{x})$, there exist a sequence of vectors $\left(x_{n}, f\left(x_{n}\right)\right) \in \mathrm{G}(f, A)$ and a sequence of positive numbers $\left\{\lambda_{n}\right\}$ such that

$$
\begin{array}{r}
\left(x_{n}, f\left(x_{n}\right)\right) \stackrel{ }{ } \longrightarrow \infty \\
\lambda_{n}\left(\left(x_{n}, f\left(x_{n}\right)\right)-(\bar{x}, f(\bar{x}))\right) \\
\longrightarrow
\end{array}
$$

It is now immediate that

$$
\begin{array}{r}
x_{n} \longrightarrow n \longrightarrow \infty \\
\lambda_{n}\left(x_{n}-\bar{x}\right) \stackrel{x}{n} x,
\end{array}
$$

and hence, by virtue of Lemma 2.1,

$$
x \in \mathrm{LC}(A, \bar{x}) .
$$


Moreover, we have

$$
\begin{aligned}
r & =\lim _{n \rightarrow \infty} \lambda_{n}\left(f\left(x_{n}\right)-f(\bar{x})\right) \\
& =\lim _{n \rightarrow \infty} \frac{f\left(\bar{x}+\frac{1}{\lambda_{n}} \lambda_{n}\left(x_{n}-\bar{x}\right)\right)-f(\bar{x})}{\frac{1}{\lambda_{n}}}=f_{\bar{x}}(x) .
\end{aligned}
$$

Consequently, we can conclude that

$$
(x, r) \in C .
$$

Conversely, let $\left(x, f_{\bar{x}}(x)\right) \in C$. Then, the fact that $x \in \operatorname{LC}(A, \bar{x})$ implies the existence of sequences $\left\{\lambda_{n} \mid \lambda_{n}>0\right\}$ and $\left\{x_{n}\right\} \subset A$ such that

$$
\begin{aligned}
x_{n} & \longrightarrow \bar{x} \quad \text { as } n \longrightarrow \infty, \\
\lambda_{n}\left(x_{n}-\bar{x}\right) & \longrightarrow x \quad \text { as } n \longrightarrow \infty .
\end{aligned}
$$

It is now clear that, by Lemma 3.1 ,

$$
\lim _{n \rightarrow \infty}\left(x_{n}, f\left(x_{n}\right)\right)=(\vec{x}, f(\vec{x})) \text {. }
$$

It is also valid that

$$
\lim _{n \rightarrow \infty} \lambda_{n}\left(f\left(x_{n}\right)-f(\bar{x})\right)=f_{\bar{x}}(x),
$$

and hence,

$$
\lim _{n \rightarrow \infty} \lambda_{n}\left(\left(x_{n}, f\left(x_{n}\right)\right)-(\bar{x}, f(\bar{x}))\right)=\left(x, f_{\bar{x}}(x)\right) .
$$

Therefore, we can claim that

$$
\left(x, f_{\bar{x}}(x)\right) \in \mathrm{LG}(f, A, \bar{x}) .
$$

DEFinition 3.2. Let be given a real valued function $f$ defined over a linear topological space $X$. Then, $f$ is called pseudoconvex over $A$ at $\bar{x}$ if $f$ is differentiable at $\bar{x}$ in the sense of Neustadt and if $x \in A$,

$$
f_{\bar{x}}(x-\bar{x}) \geqq 0 \text { implies } f(x)-f(\bar{x}) \geqq 0 .
$$

THEOREM 3.2. Let $\bar{x}$ be an optimal solution to the problem:

$$
\text { minimize }\{f(x) \mid x \in A\} \text {, }
$$

where $A$ is a set in a Banach space $X$, and $f$ is differentiable at $\bar{x}$ in the sense of Neustadt. It is then necessary that

$$
f_{\bar{x}}(x) \geqq 0 \quad \text { for all } x \in \mathrm{LC}(A, \bar{x}) .
$$

It is also sufficient if $f$ is pseudoconvex over $A$ at $\bar{x}$, and if $A$ is a pseudocone with vertex at $\bar{x}$.

Proof. Necessity: It is an immediate consequence of Theorem 3.1 and Proposition 3.1.

Sufficiency: By the definition of pseudocone, it is true that

$$
x-\bar{x} \in \operatorname{LC}(A, \bar{x}) \quad \text { for all } \quad x \in A \text {. }
$$


Hence, we have

$$
f_{\bar{x}}(x-\bar{x}) \geqq 0 \quad \text { for } \quad x \in A .
$$

By the pseudoconvexity of $f$ over $A$ at $\bar{x}$, we can conclude that

$$
f(x)-f(\bar{x}) \geqq 0 \quad \text { for all } \quad x \in A .
$$

This completes the proof of Theorem 3.2.

In order to obtain the necessary conditions to nonlinear programming, we introduce the following definitions.

Definition 3.3. Let $A$ and $H$ be arbitrary subsets of a linear topological space $X$, and let $\bar{x} \in A \cap H$. Then, the subset $A$ is called to be locally relatively convex with respect to $H$ at $\bar{x}$ if there exists a neighborhood $N$ of $\bar{x}$ in $X$ such that

$$
\operatorname{co}(A) \cap H \cap N=A \cap H \cap N .
$$

DEFinition 3.4. Let $A$ be an arbitrary subset of a locally convex linear topological space $X$ and let $\bar{x} \in A$. Then, $A$ is called to be locally convex at $\bar{x}$ if there exists a convex neighborhood $N$ of $\bar{x}$ in $X$ such that the set $A \cap N$ is convex.

LEMMA 3.2. If $A$ is locally relatively convex with respect to $H$ at $\bar{x}$, then it is valid that

$$
\mathrm{LC}(A \cap H, \bar{x})=\mathrm{LC}(\operatorname{co}(A) \cap H, \bar{x}) .
$$

Proof. Since $A$ is locally relatively convex with respect to $H$ at $\bar{x}$, there exists a neighborhood $\tilde{N}$ of $\bar{x}$ such that

Then, we have

$$
\operatorname{co}(A) \cap H \cap \tilde{N}=A \cap H \cap \tilde{N} .
$$

$$
\begin{aligned}
\mathrm{LC}(\operatorname{co}(A) \cap H, \bar{x}) & =\bigcap_{\substack{N \in \Re(\bar{x})\\
}} \mathrm{C}(\operatorname{co}(A) \cap H \cap N, \bar{x}) \\
& =\overbrace{\substack{N \subset \tilde{N} \\
N \in \mathscr{N}(\bar{x})}} \mathrm{C}(\operatorname{co}(A) \cap H \cap N, \bar{x}) \\
& =\bigcap_{\substack{N \subset \tilde{N} \\
N=\Re(\bar{x})}} \mathrm{C}(A \cap H \cap N, \bar{x}) \\
& =\operatorname{LC}(A \cap H, \bar{x}) .
\end{aligned}
$$

Now, we shall consider the following nonlinear programming in a Banach space. Let $X$ and $Y$ be real Banach spaces, $A$ an arbitrary subset of $X, B$ a closed convex cone with vertex at the origin having a non-empty interior, $f$ a real valued function defined over $X$, and $g$ a mapping of $X$ into $Y$. Then, find a vector $\bar{x} \in A$ satisfying

$$
\begin{array}{ll}
\text { minimize } & f(x) \\
\text { subject to } & x \in A \\
& g(x) \in B .
\end{array}
$$

DEFinition 3.5. Let $B$ be a closed convex cone with vertex at the origin in $Y$, and $h$ a mapping of $X$ into $Y$. Then, $h$ is called to be $B$-convex if $\left[h\left(\alpha x_{1}+\beta x_{2}\right)\right.$ $\left.-\alpha h\left(x_{1}\right)-\beta h\left(x_{2}\right)\right] \in B$ whenever $(\alpha, \beta) \in P^{2}$ and $x_{1}, x_{2} \in X$.

We can now describe our main theorems. 
THEOREM 3.3. Let $\bar{x} \in A$ be the optimal solution to the problem $(P)$. Assume that

(a) $A$ is locally convex at $\bar{x}$, or is locally relatively convex with respect to $H=g^{-1}(B)=\{x \in X \mid g(x) \in B\}$ at $\bar{x}$.

(b) the functions $f$ and $g$ are differentiable at $\bar{x}$ in the sense of Neustadt, the differential $f_{\bar{x}}(x)$ is convex continuous in $x$ and the differential $g_{\bar{x}}(x)$ is $B$ convex continuous in $x$.

Then there exist a real number $\eta$ and a linear continuous functional $\bar{y}^{*} \in Y^{*}$, not both zero, such that

$$
\begin{gathered}
\eta \geqq 0, \\
\bar{y}^{*}(y) \leqq 0 \quad \text { for all } y \in B, \\
\bar{y}^{*}(g(\bar{x}))=0, \\
g(\bar{x}) \in B, \\
\eta f_{\bar{x}}(x)+\bar{y}^{*}\left(g_{\bar{x}}(x)\right) \geqq 0 \quad \text { for all } x \in \mathrm{P}(A, \bar{x}) .
\end{gathered}
$$

PROOF. Let

$$
E=\left\{\left(g_{\bar{x}}(x), f_{\bar{x}}(x)\right)-(b, r) \in Y \times R^{1} \mid x \in \mathrm{P}(A, \bar{x}), b \in B, r \leqq 0\right\} .
$$

Then it is clear that $E$ is a convex subset of $Y \times R^{1}$ by virtue of the convexity of $g_{\bar{x}}$ and $f_{\bar{x}}$.

Furthermore, define the subset $\tilde{B}$ of $Y \times R^{1}$ as follows:

$$
\tilde{B}=\{\tilde{b} \in Y \mid \tilde{b}=b-g(\bar{x}), b \in \operatorname{int} B\} \times\left\{r \in R^{1} \mid r<0\right\} . *
$$

It is immediate that $\tilde{B}$ is an open convex cone in $Y \times R^{1}$.

We shall show that

$$
E \cap \tilde{B}=\emptyset \text {. }
$$

To show the contradiction, assume that there exists a vector $(y, \alpha) \in Y \times R^{1}$ such that

$$
(y, \alpha) \in E \cap \tilde{B} .
$$

Then there are vectors $b_{1} \in \operatorname{int} B, b_{2} \in B, x \in \mathrm{P}(A, \bar{x})$ and a real number $r \leqq 0$ such that

$$
(y, \alpha)=\left(b_{1}-g(\bar{x}), \alpha\right)=\left(\left(g_{\bar{x}}(x), f_{\bar{x}}(x)\right)-\left(b_{2}, r\right)\right),
$$

or

$$
\begin{aligned}
& g_{\bar{x}}(x)+g(\bar{x})=b_{1}+b_{2}, \\
& f_{\bar{x}}(x) \leqq f_{\bar{x}}(x)-r=\alpha<0 .
\end{aligned}
$$

It follows from Lemma 3.1 in [5] that

$$
g_{\bar{x}}(x)+g(\bar{x}) \in \operatorname{int} B,
$$

so that there exists a neighborhood $U$ of the origin in $Y$ such that

$$
g_{\bar{x}}(x)+g(\bar{x})+U \subset \operatorname{int} B .
$$

Since $x \in P(A, \bar{x})$, it follows from Lemma 2.2 that there are a sequence of posi-

* If $B$ is a subset of a topological space, then int $B$ denotes the interior of $B$. 
tive numbers $\left\{\lambda_{n}\right\}$, a sequence of integers $\left\{m_{n}\right\}$, a sequence of vectors $\left(\alpha_{n 1}, \cdots, \alpha_{n m_{n}}\right)$ $\in P^{m_{n}}$, and a sequence of vectors $\left\{x_{n i}\right\}_{i=1, \cdots, m_{n}} \subset A$ such that

$$
\begin{aligned}
& x_{n i} \longrightarrow n \longrightarrow \infty \bar{x} \quad \text { uniformly in } i, \\
& \lambda_{n}\left(\sum_{i=1}^{m_{n}} \alpha_{n i} x_{n i}-\bar{x}\right) \stackrel{ }{\longrightarrow \longrightarrow \infty} x .
\end{aligned}
$$

Then, we have

$$
\begin{aligned}
& \lambda_{n}\left[g\left(\sum_{i=1}^{m_{n}} \alpha_{n i} x_{n i}\right)-g(\bar{x})\right] \\
= & \frac{g\left(\bar{x}+\frac{1}{\lambda_{n}} \cdot \lambda_{n}\left(\sum_{i} \alpha_{n i} x_{n i}-\bar{x}\right)\right)-g(\bar{x})}{\frac{1}{\lambda_{n}}}-\frac{}{n \longrightarrow \infty} g_{\bar{x}}(x),
\end{aligned}
$$

and hence there is a positive integer $M_{1}$ such that

$$
\lambda_{n}\left[g\left(\sum_{i=1}^{m_{n}} \alpha_{n i} x_{n i}\right)-g(\bar{x})\right] \in g_{\bar{x}}(x)+U \quad \text { whenever } n \geqq M_{1} .
$$

Since $\lambda_{n} \rightarrow \infty$ as $n \rightarrow \infty$, there is a positive integer $M_{2}$ satisfying $\lambda_{n}>1$ for $n \geqq M_{2}$. Let $M=\max \left\{M_{1}, M_{2}\right\}$. For each $n \geqq M$, it is valid that

$$
\begin{aligned}
\lambda_{n} g\left(\sum_{i=1}^{m_{n}} \alpha_{n i} x_{n i}\right) & \in g_{\bar{x}}(x)+\lambda_{n} g(\bar{x})+U \\
& \subset \lambda_{n} g(\bar{x})-g(\bar{x})+\operatorname{int} B \\
& =\left(\lambda_{n}-1\right) g(\bar{x})+\operatorname{int} B \subset \operatorname{int} B \subset B,
\end{aligned}
$$

so that

$$
\sum_{i=1}^{m_{n}} \alpha_{n i} x_{n i} \in H \quad \text { for all } n \geqq M .
$$

If $A$ is locally convex at $\bar{x}$, then for sufficiently large $M^{\prime}$ we have

$$
\sum_{i=1}^{m_{n}} \alpha_{n i} x_{n i} \in H \cap A \quad \text { for all } n \geqq M^{\prime},
$$

and, by virtue of Lemma 2.1 , we obtain

$$
x \in \mathrm{LC}(A \cap H, \tilde{x}) .
$$

It then follows from Theorem 3.1 and Proposition 3.1 that

$$
f_{\bar{x}}(x) \geqq 0,
$$

which contradicts to (3.6).

If $A$ is locally relatively convex with respect to $H$ at $\bar{x}$, then we have, by virtue of Lemma 3.2,

$$
x \in \mathrm{LC}(\operatorname{co}(A) \cap H, \bar{x})=\mathrm{LC}(A \cap H, \bar{x}) .
$$

Now, it follows from Theorem 3.1 and Proposition 3.1 that

which contradicts to (3.6).

$$
f_{\bar{x}}(x) \geqq 0,
$$


Consequently, we can conclude that

$$
E \cap \hat{B}=\emptyset,
$$

if $A$ is locally convex at $\bar{x}$, or if $A$ is locally relatively convex with respect to $H$ at $\bar{x}$. Now, it follows, on the basis of the separation theorem in a linear topological space (Theorem V.2.8 in [2]), that there is a non-zero continuous linear functional $z^{*} \in\left(Y \times R^{1}\right) *$ such that

$$
z^{*}\left(\left(y_{1}, \alpha_{1}\right)\right) \leqq 0 \leqq z^{*}\left(\left(y_{2}, \alpha_{2}\right)\right) \quad \text { for all }\left(y_{1}, \alpha_{1}\right) \in \tilde{B} \text { and all }\left(y_{2}, \alpha_{2}\right) \in E \text {. }
$$

It is true that there are a continuous linear functional $\bar{y}^{*} \in Y^{*}$ and a real number $\eta$ such that

$$
z^{*}((y, \alpha))=\bar{y}^{*}(y)+\eta \alpha \quad \text { for all } \quad(y, \alpha) \in Y \times R^{1},
$$

and so

$$
\begin{array}{r}
\bar{y}^{*}\left(y_{1}-g(\bar{x})\right)+\eta \alpha_{1} \leqq 0 \leqq \bar{y}^{*}\left(g_{\bar{x}}(x)-b\right)+\eta\left(f_{\bar{x}}(x)-r\right) \quad \text { for all } y_{1} \in \operatorname{int} B, \\
\text { all } \alpha_{1}<0, \text { all } x \in \mathrm{P}(A, \bar{x}), \text { all } b \in B \text { and all } r \leqq 0 .
\end{array}
$$

Since $0 \in \mathrm{P}(A, \bar{x})$ and $0 \in B$, we have

Furthermore,

$$
\begin{aligned}
& \eta \geqq 0, \\
& \bar{y}^{*}(b) \leqq 0 \quad \text { for all } \quad b \in B .
\end{aligned}
$$

$$
\bar{y}^{*}\left(g_{\bar{x}}(x)\right)+\eta f_{\bar{x}}(x) \geqq 0 \quad \text { for all } \quad x \in \mathrm{P}(A, \bar{x}) .
$$

Finally, it is valid that

$$
\bar{y}^{*}(g(\bar{x}))=0,
$$

since $2 g(\bar{x}) \in \overline{\operatorname{int} B}$. This completes the proof of Theorem 3.3.

REMARK : As an example of the function $f$ satisfying the hypothesis in Theorem 3.3, consider the following one: Let $c(t)=\left(c_{1}(t), \cdots, c_{n}(t)\right)$ be a continuous function from the compact interval $I=[a, b]$ into $n$-Eucledian space $R^{n}$ and $F$ a differentiable function (in the ordinary sence) from $R^{1}$ into $R^{1}$. Then, define the function $f$ from $R^{n}$ into $R^{1}$ by

$$
f(x)=\int_{a}^{b} F(\|x-c(t)\|) d t \quad \text { for all } \quad x \in R^{n},
$$

where $\|\cdot\|$ denotes the Eucledian norm. The differential $f_{\bar{x}}(x)$ at $\bar{x} \in R^{n}$ in the sense of Neustadt takes the form:

$$
f_{\bar{x}}(x)=\int_{I \backslash I_{0}} F^{\prime}(\|\bar{x}-c(t)\|) \frac{\sum x_{i}\left(\bar{x}_{i}-c_{i}(t)\right)}{2\|\bar{x}-c(t)\|} d t+\int_{I_{0}} F^{\prime}(\|\bar{x}-c(t)\|) \cdot\|x\| d t,
$$

where $F^{\prime}$ denotes the derivative of $F$ and

Hence, if

$$
I_{0}=\{t \in[a, b] \mid \bar{x}=c(t)\} .
$$

$$
\int_{I_{0}} F^{\prime}(\|\bar{x}-c(t)\|) d t \geqq 0,
$$

then the differential $f_{\bar{x}}(x)$ is convex on $R^{n}$. 
THEOREM 3.4. Let the notations in Theorem 3.3 still hold. Suppose that $f$ and $g$ are differentiable at $\bar{x}$ in the sense of Neustadt. Then, in order that the vector $\bar{x} \in A$ is an optimal solution to the problem $(P)$, the conditions (3.1)-(3.5) in Theorem 3.3 are sufficient if $A \cap H$ is a pseudocone with vertex at $\bar{x}$, if $f$ is pseudoconvex over $A \cap H$ at $\bar{x}$, and if $\eta \neq 0$.

Proof. First of all, (3.4) implies $\bar{x} \in A \cap H$. Since $A \cap H$ is a pseudocone with vertex at $\bar{x}$, we have

$$
x-\bar{x} \in \mathrm{LC}(A \cap H, \bar{x}) \subset \mathrm{P}(A, \bar{x}) \cap \mathrm{LC}(H, \bar{x}) \quad \text { for all } \quad x \in A \cap H .
$$

We shall show that

$$
\bar{y}^{*}\left(g_{\bar{x}}(x)\right) \leqq 0 \quad \text { for all } \quad x \in \mathrm{LC}(H, \bar{x}) .
$$

For each $x \in \operatorname{LC}(H, \bar{x})$, there exist a sequence of positive numbers $\left\{\lambda_{n}\right\}$, and a sequence of vectors $\left\{x_{n}\right\} \subset H$ such that

$$
\begin{aligned}
x_{n} \longrightarrow \bar{x} & \text { as } n \longrightarrow \infty, \\
\lambda_{n}\left(x_{n}-\bar{x}\right) \longrightarrow x & \text { as } n \longrightarrow \infty .
\end{aligned}
$$

Note that

$$
g_{\bar{x}}(x)=\lim _{n \rightarrow \infty} \lambda_{n}\left[g\left(x_{n}\right)-g(\bar{x})\right]
$$

Therefore, we can obtain

$$
\begin{aligned}
\bar{y}^{*}\left(g_{\bar{x}}(x)\right) & =\bar{y}^{*}\left(\lim _{n \rightarrow \infty} \lambda_{n}\left[g\left(x_{n}\right)-g(\bar{x})\right]\right) \\
& =\lim _{n \rightarrow \infty} \bar{y}^{*}\left(\lambda_{n}\left[g\left(x_{n}\right)-g(\bar{x})\right]\right) \\
& =\lim _{n \rightarrow \infty} \lambda_{n}\left(\bar{y}^{*}\left(g\left(x_{n}\right)\right)-\bar{y}^{*}(g(\bar{x}))\right)
\end{aligned}
$$

since $\bar{y}^{*}$ is a continuous linear functional. It follows from (3.2) and (3.3) that

and hence

$$
\lambda_{n}\left(\bar{y}^{*}\left(g\left(x_{n}\right)\right)-\bar{y}^{*}(g(\bar{x}))\right) \leqq 0 \quad \text { for all } n,
$$

$$
\bar{y}^{*}\left(g_{\bar{x}}(x)\right) \leqq 0 \quad \text { for all } \quad x \in \operatorname{LC}(H, \bar{x}) .
$$

By virtue of (3.5), we have

$$
\eta f_{\bar{x}}(x) \geqq-\bar{y}^{*}\left(g_{\bar{x}}(x)\right) \geqq 0 \quad \text { for all } \quad x \in \mathrm{P}(A, \bar{x}) \cap \mathrm{LC}(H, \bar{x}) .
$$

Since $x-\bar{x} \in \mathrm{P}(A, \bar{x}) \cap \mathrm{LC}(H, \bar{x})$ for all $x \in A \cap H$, it is valid that

$$
\eta f_{\bar{x}}(x-\bar{x}) \geqq 0 \quad \text { for all } \quad x \in A \cap H .
$$

The hypothesis and (3.1) implies $\eta>0$, and therefore

$$
f_{\bar{x}}(x-\bar{x}) \geqq 0 \quad \text { for all } \quad x \in A \cap H .
$$

Since $f$ is pseudoconvex over $A \cap H$ at $\bar{x}$, we obtain

$$
f(x)-f(\bar{x}) \geqq 0 \quad \text { for all } x \in A \cap H .
$$

Consequently, we can conclude that the vector $\bar{x}$ is a minimum over $A \cap H$. This completes the proof of Theorem 3.4. 
Let us generalize Theorem 3.4, since there is the example which does not fall into the category of this theorem. Indeed, consider the following example which was introduced by Zlobec [9]:

$$
\begin{aligned}
& X=A=R^{2}, \quad Y=R^{3}, \\
& B=\left\{\left(y_{1}, y_{2}, y_{3}\right) \mid 2 y_{1} y_{3} \geqq y_{2}^{2}, y_{1} \geqq 0, y_{3} \geqq 0\right\}, \\
& g_{1}\left(x_{1}, x_{2}\right)=-x_{1}^{2}, \\
& g_{2}\left(x_{1}, x_{2}\right)=x_{1}, \\
& g_{3}\left(x_{1}, x_{2}\right)=x_{2}, \\
& f\left(x_{1}, x_{2}\right)=x_{1}+x_{2}^{2} .
\end{aligned}
$$

Let $\bar{x}=0$, then $\mathrm{P}(A, \bar{x})=R^{2}$. We have

$$
f_{\bar{x}}(x)=x_{1}, \quad g_{\bar{x}}(x)=\left(\begin{array}{c}
0 \\
x_{1} \\
x_{2}
\end{array}\right) .
$$

Then, the real number $\eta$ and the linear continuous functional $\bar{y}^{*} \in Y^{*}$ satisfying (3.1)-(3.5) turn out to be:

$$
\eta=0, \quad \bar{y}^{*}=\left(\begin{array}{l}
\lambda \\
0 \\
0
\end{array}\right) \quad \text { for all } \lambda<0 .
$$

Hence, Theorem 3.4 can not be applied to this example. On the other hand, Theorem 3.3 is available.

THEOREM 3.5. Let the notations in Theorem 3.3 still hold and let $f$ and $g$ be differentiable at $\bar{x}$ in the sence of Neustadt. If there exist a vector $\bar{x} \in A$, a real number $\eta$, and a sequense of linear continuous functionals $\left\{\bar{y}_{i}^{*}\right\} \subset Y^{*}$ such that

$$
\begin{gathered}
\eta>0, \\
\bar{y}_{i}^{*}(y) \leqq 0 \quad \text { for all } y \in B, i=1,2, \cdots, \\
\bar{y}_{i}^{*}(g(\bar{x}))=0 \quad \text { for } i=1,2, \cdots, \\
\qquad g(\bar{x}) \in B, \\
\lim _{i \rightarrow \infty}\left[\eta f_{\bar{x}}(x)+\bar{y}_{i}^{*}\left(g_{\bar{x}}(x)\right)\right] \geqq 0 \quad \text { for all } x \in \mathrm{P}(A, \bar{x}),
\end{gathered}
$$

then the vector $\bar{x}$ is an optimal solution to the problem $(P)$ if $A \cap H$ is a pseudocone with vertex at $\bar{x}$ and if $f$ is pseudoconvex over $A \cap H$ at $\bar{x}$.

PROOF. It follows from the proof of Theorem 3.4 that

$$
x-\bar{x} \in \mathrm{LC}(A \cap H, \bar{x}) \subset \mathrm{P}(A, \bar{x}) \cap \mathrm{LC}(H, \bar{x}) \quad \text { for all } \quad x \in A \cap H,
$$

and that

$$
y_{i}^{*}\left(g_{\bar{x}}(x)\right) \leqq 0 \quad \text { for all } \quad x \in \mathrm{LC}(H, \bar{x})
$$

Hence,

$$
\eta f_{\bar{x}}(x)+\bar{y}_{i}^{*}\left(g_{\bar{x}}(x)\right) \leqq \eta f_{\bar{x}}(x) \quad \text { for } \quad i=1,2, \cdots .
$$


By virtue of (3.11), we have

$$
0 \leqq \lim _{i \rightarrow \infty}\left[\eta f_{\bar{x}}(x)+\bar{y}_{i}^{*}\left(g_{\bar{x}}(x)\right)\right] \leqq \eta f_{\bar{x}}(x) \quad \text { for all } \quad x \in \mathrm{P}(A, \bar{x}) \cap \operatorname{LC}(H, \bar{x}) .
$$

Therefore, it is true, by (3.7), that

$$
f_{\bar{x}}(x-\bar{x}) \geqq 0 \quad \text { for all } \quad x \in A \cap H .
$$

The pseudoconvexity of $f$ implies that the vector $\bar{x}$ is an optimal solution to the problem $(\mathrm{P})$. This completes the proof of Theorem 3.5.

It is easily verified that Theorem 3.5 is more general than Theorem 3.4 and that the preceding example is contained in Theorem 3.5 with $\bar{y}_{i}^{*}=\left(\begin{array}{c}i \\ 1 \\ 1 / 2 i\end{array}\right), i=1,2, \cdots$.

Let the notations in Theorem 3.3 still hold. We now consider the following problems.

$\left(\mathrm{P}^{\prime}\right)$ Find $\bar{x} \in A$ and $\bar{y}^{*} \in B^{-}$such that

$$
\phi\left(\bar{x}, y^{*}\right) \leqq \phi\left(\bar{x}, \bar{y}^{*}\right) \leqq \phi\left(x, \bar{y}^{*}\right) \quad \text { for all } \quad x \in A \text { and all } y^{*} \in B^{-},
$$

where $\phi\left(x, y^{*}\right)=f(x)+y^{*}(g(x))$.

$\left(\mathrm{P}^{\prime \prime}\left(\bar{y}^{*}\right)\right)$ Find $\bar{x}$ which (for fixed $\bar{y}^{*}$ ) solves:

$$
\text { minimize }\left\{f(x)+\bar{y}^{*}(g(x)) \mid x \in A\right\} .
$$

THEOREM 3.6. (i) Let $\left(\bar{x}, \bar{y}^{*}\right)$ be the optimal solution to the problem $\left(P^{\prime}\right)$. Then, the conditions

$$
\begin{gathered}
f_{\bar{x}}(x)+\bar{y}^{*}\left(g_{\bar{x}}(x)\right) \geqq 0 \quad \text { for all } x \in \operatorname{LC}(A, \bar{x}), \\
\bar{y}^{*}(g(\bar{x}))=0, \\
g(\bar{x}) \in B,
\end{gathered}
$$

hold.

(ii) If $A$ is a pseudocone with vertex at $\bar{x}$, and if $\phi\left(x, \bar{y}^{*}\right)$ is pseudoconvex over $A$ at $\bar{x}$, then the conditions (3.12)-(3.14) are sufficient for $\left(\bar{x}, \bar{y}^{*}\right)$ to solve $\left(P^{\prime}\right)$.

(iii) If $\left(\bar{x}, \bar{y}^{*}\right)$ solves $\left(P^{\prime}\right)$, then $\bar{x}$ solves $(P)$.

Proof. The condition (3.14) follows from Lemma 5.1 in the sequel. The other conditions are easily verified.

Definition 3.5. The set $A$ is called to be pseudoconvex at $\bar{x}(\in A)$ if

$$
x-\bar{x} \in \mathrm{P}(A, \bar{x}) \quad \text { for all } x \in A .
$$

Note that if the set $A$ is a pseudocone with vertex at $\bar{x}$, then it is pseudoconvex at $\bar{x}$.

THEOREM 3.7. If $\bar{x}$ solves $(P)$, and if the hypotheses in Theorem 3.3 are satisfied, then $\left(\bar{x}, \frac{1}{\eta} \bar{y}^{*}\right)$ solves $\left(P^{\prime}\right)$ if $\eta \neq 0$, if $\phi\left(x, \frac{1}{\eta} \bar{y}^{*}\right)$ is pseudoconvex over $A$ at $\bar{x}$, and if $A$ is pseudoconvex at $\bar{x}$.

Proof. Since $\bar{x}$ solves $(P)$, it follows from Theorem 3.3 that the conditions (3.1)-(3.5) hold. Since $A$ is pseudoconvex at $\bar{x}$, the conditions (3.1) and (3.5) imply 


$$
\phi_{\bar{x}}\left(x-\bar{x}, \frac{1}{\eta} \bar{y}^{*}\right)=f_{\bar{x}}(x-\bar{x})+\frac{1}{\eta} \bar{y}^{*}\left(g_{\bar{x}}(x-\bar{x})\right) \geqq 0 \quad \text { for all } \quad x \in A .
$$

Since $\phi\left(x, \frac{1}{\eta} \bar{y}^{*}\right)$ is pseudoconvex over $A$ at $\bar{x}$, we have

$$
\phi\left(x, \frac{1}{\eta} \bar{y}^{*}\right)-\phi\left(\bar{x}, \frac{1}{\eta} \bar{y}^{*}\right) \geqq 0 \quad \text { for all } \quad x \in A .
$$

By virtue of (3.2), (3.3) and (3.4), it is valid that

$$
\phi\left(\bar{x}, y^{*}\right) \leqq \phi\left(\bar{x}, \frac{1}{\eta} \bar{y}^{*}\right) \quad \text { for all } y^{*} \in B^{-} .
$$

Therefore, $\left(\bar{x}, \frac{1}{\eta} \bar{y}^{*}\right)$ solves $\left(P^{\prime}\right)$.

THEOREM 3.8. (i) Let $\bar{x}$ solve $P^{\prime \prime}\left(\bar{y}^{*}\right)$. Then, it is true that

$$
f_{\bar{x}}(x)+\bar{y}^{*}\left(g_{\bar{x}}(x)\right) \geqq 0 \quad \text { for all } \quad x \in \mathrm{LC}(A, \bar{x}) \text {. }
$$

(ii) If in addition, $\bar{y}^{*} \in B^{-}$, then $\left(\bar{x}, \bar{y}^{*}\right)$ solves $\left(P^{\prime}\right)$ if and only if

$$
g(\bar{x}) \in B \quad \text { and } \quad \bar{y}^{*}(g(\bar{x}))=0 .
$$

Proof. These are immediate consequences of Theorems 3.2 and 3.6.

\section{An application to convex programming in a Banach space.}

In this section, we shall present the necessary and sufficient conditions for an ordinary convex program in a Banach space.

First of all, we can verify the following proposition.

PROPOSITION 4.1. Let be given a convex function $f$ defined on an open convex subset $E$ of a real linear topological space $X$. If $f$ is bounded above on some neighborhood of a point in $E$, then $f$ is differentiable over $E$ in the sense of Neustadt.

PRoof. Note that, by virtue of Proposition 21 in [1] (Ch. II, $\left.\S 2, \mathrm{n}^{\circ} 10\right), f$ is continuous on $E$. We shall snow that the directional derivative $\delta f(\bar{x} ; x)$ at $\bar{x} \in E$ defined by

$$
\delta f(\bar{x} ; x)=\lim _{\lambda \rightarrow 0+} \frac{f(\bar{x}+\lambda x)-f(\bar{x})}{\lambda},
$$

is continuous in $x$ on $X$. Since $\delta f(\bar{x} ; x)$ is a convex function defined over $X$, it is sufficient to show that $\delta f(\bar{x} ; x)$ is bounded above on a neighborhood of a point, say, the origin. But this is an immediate consequence of the continuity of $f$ and the fact that

$$
\delta f(\bar{x} ; x) \leqq \frac{f(\bar{x}+\varepsilon x)-f(\bar{x})}{\varepsilon} \leqq f(\bar{x}+x)-f(\bar{x}) \quad \text { for all } \quad \varepsilon, 0<\varepsilon<1 .
$$

Now, we shall prove the proposition. For every $x, y \in X$ and $0<\varepsilon<1$, we have

$$
\frac{f(\bar{x}+\varepsilon y)-f(\bar{x})}{\varepsilon}-\delta f(\bar{x} ; x) \geqq \delta f(\bar{x} ; y)-\delta f(\bar{x} ; x),
$$

and, moreover, 


$$
\begin{aligned}
& \frac{f(\bar{x}+\varepsilon y)-f(\bar{x})}{\varepsilon}-\delta f(\bar{x} ; x) \\
= & \frac{f(\varepsilon \bar{x}+\varepsilon(y-x)+(1-\varepsilon) \bar{x}+\varepsilon x)-f(\bar{x})}{\varepsilon}-\delta f(\bar{x} ; x) \\
\leqq & \frac{\varepsilon f(\bar{x}+(y-x))+(1-\varepsilon) f\left(\bar{x}+\frac{\varepsilon}{1-\varepsilon} x\right)-f(\bar{x})}{\varepsilon}-\delta f(\bar{x} ; x) \\
= & f(\bar{x}+(y-x))-f(\bar{x})+\frac{f\left(\bar{x}+\frac{\varepsilon}{1-\varepsilon} x\right)-f(\bar{x})}{\frac{\varepsilon}{1-\varepsilon}}-\delta f(\bar{x} ; x) .
\end{aligned}
$$

Since $\delta f(\bar{x} ; y)$ and $f(y)$ are continuous, we conclude that

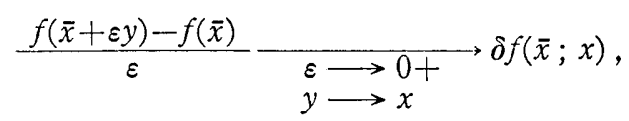

which implies that $f$ is differentiable in the sense of Neustadt and that the differential equals to the directional derivative $\delta f(\bar{x} ; x)$.

It is now easily seen that, under the same hypotheses of the above proposition, the convex function $f$ is pseudoconvex at each $\bar{x} \in E$.

THEOREM 4.1. Let $f, g_{1}, \cdots, g_{n}$ be all real valued convex functions defined over an open, convex subset $E$ of a Banach space $X$. Assume that all $f, g_{1}, \cdots, g_{n}$ are bounded above on a neighborhood of a point in $E$. Given a convex subset $A$ of $E$, consider the following convex programming:

$$
\begin{array}{ll}
\text { minimize } & f(x) \\
\text { subject to } & x \in A \\
& g_{i}(x) \leqq 0, \quad i=1, \cdots, n .
\end{array}
$$

Assume that there is at least one point $x \in A$ satisfying $g_{i}(x)<0$, for $i=1, \cdots, n$. In order that $\bar{x} \in A$ is an optimal solution, it is necessary and sufficient that there exist non-negative numbers $\lambda_{0}, \lambda_{1}, \cdots, \lambda_{n}$, not all zero, such that

$$
\lambda_{0} \delta f(\bar{x} ; x)+\lambda_{1} \delta g_{1}(\bar{x} ; x)+\cdots+\lambda_{n} \delta g_{n}(\bar{x} ; x) \geqq 0 \quad \text { for all } x \in P(A, \bar{x}) .
$$

Proof. Necessity: Since $A$ is convex, it is clear that $A$ is locally convex at any point in $A$. It follows from Proposition 4.1 that $f, g_{1}, \cdots, g_{n}$ are all differentiable at $\bar{x}$ in the sense of Neustadt and that their differentials are coincide with $\delta f(\bar{x} ; x)$, $\delta g_{1}(\bar{x} ; x), \cdots, \delta g_{n}(\bar{x} ; x)$, respectively. It is valid that if we define the closed convex cone $B$ in $R^{n}$ by

$$
B=\left\{\left(y_{1}, \cdots, y_{n}\right) \in R^{n} \mid y_{i} \leqq 0, i=1, \cdots, n\right\},
$$

then $g=\left(g_{1}, \cdots, g_{n}\right)$ is $B$-convex and has the differential $g_{\bar{x}}(x)=\left(\delta g_{1}(\bar{x} ; x), \cdots, \delta g_{n}(\bar{x} ; x)\right)$. Therefore, we can apply Theorem 3.3 to this problem, so that there are a real number $\lambda_{0}$ and a vector $\lambda=\left(\lambda_{1}, \cdots, \lambda_{n}\right) \in\left(R^{n}\right)^{*}=R^{n}$, not both zero, such that 


$$
\begin{gathered}
\quad \lambda_{0} \geqq 0, \\
\lambda \cdot y \leqq 0 \quad \text { for all } y \in B, \\
\lambda \cdot g(\bar{x})=0, \\
g(\bar{x}) \in B, \\
\lambda_{0} f_{\bar{x}}(x)+\lambda \cdot g_{\bar{x}}(x) \geqq 0 \quad \text { for all } \quad x \in P(A, \bar{x}) .
\end{gathered}
$$

By virtue of (4.1) and (4.2), it is immediate that $\lambda_{i} \geqq 0$ for $i=1, \cdots, n$. It then follows from (4.3) and (4.4) that $\lambda_{i} g_{i}(\bar{x})=0$ for $i=1, \cdots, n$. Since $f_{\bar{x}}(x)=\delta f(\bar{x} ; x)$, (c) holds by (4.5).

Now, let us verify (a). Since $A$ is convex, it is clear that

$$
x-\bar{x} \in P(A, \bar{x}) \quad \text { for all } \quad x \in A \text {. }
$$

By virtue of (4.5), we have

$$
\lambda_{0} \delta f(\bar{x} ; x-\bar{x})+\lambda_{1} \delta g_{1}(\bar{x} ; x-\bar{x})+\cdots+\lambda_{n} \delta g_{n}(\bar{x} ; x-\bar{x}) \geqq 0 \quad \text { for all } \quad x \in A .
$$

From the property of convex functions follow

and hence

$$
\begin{aligned}
& f(x)-f(\bar{x}) \geqq \delta f(\bar{x} ; x-\bar{x}), \\
& g_{i}(x)-g_{i}(\bar{x}) \geqq \delta g_{i}(\bar{x} ; x-\bar{x}),
\end{aligned}
$$

$$
\begin{aligned}
& \lambda_{0} f(x)+\lambda_{1} g_{1}(x)+\cdots+\lambda_{n} g_{n}(x) \\
\geqq & \lambda_{0} f(\bar{x})+\lambda_{1} g_{1}(\bar{x})+\cdots+\lambda_{n} g_{n}(\bar{x})=\lambda_{0} f(\bar{x}),
\end{aligned}
$$

To show the contradiction suppose that $\lambda_{0}=0$. Then, we have

$$
\lambda_{1} g_{1}(x)+\cdots+\lambda_{n} g_{n}(x) \geqq 0 \quad \text { for all } x \in A .
$$

By the hypothesis, there is a vector $x_{0} \in A$ such that $g_{i}\left(x_{0}\right)<0$ for $i=1, \cdots, n$, and hence $\lambda_{i}=0$ for $i=1, \cdots, n$, which is a contradiction.

Sufficiency: Let $H=g^{-1}(B)$. Since $A \cap H$ is convex, it is a pseudocone with vertex at $\bar{x}(\in A \cap H)$. Moreover, since $f$ is convex, it is a pseudoconvex over $A \cap H$ at $\bar{x}$. It now follows from Theorem 3.4 that $\bar{x}$ is an optimal solution.

\section{On constraint qualification.}

To begin with, we shall state the following lemma.

LEMMA 5.1 (cf. Guignard [3]). Let $X$ be a locally convex linear topological space and let $A$ be a cone in $X$. If the vector $x \in X$ satisfies

then we have

$$
a^{*}(x) \leqq 0 \quad \text { for all } a^{*} \in A^{-},
$$

$$
x \in \overline{\operatorname{co}(A)} .
$$

LEMMA 5.2. Let $A$ and $B$ be cones in a locally convex linear topological space $X$. If $\tilde{x}^{*} \in X^{*}$ is a continuous linear functional satisfying

then it is true that

$$
\tilde{x}^{*}(x) \leqq 0 \quad \text { for all } x \in \overline{\operatorname{co}(A)} \cap \overline{\operatorname{co}(B)},
$$




$$
\tilde{x}^{*} \in \overline{A^{-}+B^{-}} .
$$

Proof. Let $\tilde{x}^{*} \in X^{*}$ satisfy the condition in the lemma. To show the contradiction assume that

$$
\tilde{x}^{*} \notin \overline{A^{-}+B^{-}} .
$$

Since $\overline{A^{-}+B^{-}}$is a $X$-closed convex cone in $X^{*}$, it follows from the separation theorem that there is a non-zero element $\tilde{x} \in X$ such that

$$
x^{*}(\tilde{x}) \leqq 0<\tilde{x}^{*}(\tilde{x}) \quad \text { for all } \quad x^{*} \in \overline{A^{-}+B^{-}} .
$$

Then, by virtue of Lemma 5.1 and the fact that $A^{-}, B^{-} \subset \overline{A^{-}+B^{-}}$, it is easily verified that

and hence

$$
\tilde{x} \in \overline{\operatorname{co}(A)} \cap \overline{\operatorname{co}(B)} .
$$

which contradicts to (5.1).

$$
\tilde{x}^{*}(\tilde{x}) \leqq 0,
$$

Now, we can present the following proposition.

PROPOSITION 5.1. Let $A$ and $B$ be cones in a locally convex linear topological space $X$. Then, the condition

holds if and only if

$$
(A \cap B)^{-}=\overline{A^{-}+B^{-}}
$$

$$
\overline{\operatorname{co}(A \cap \bar{B})}=\overline{\operatorname{co}(A)} \cap \overline{\operatorname{co}(B)} .
$$

Proof. "if" part: Since

$$
A^{-}+B^{-} \subset(A \cap B)^{-},
$$

and since $(A \cap B)^{-}$is $X$-closed, it is clear that

$$
\overline{A^{-}+B^{-}} \subset(A \cap B)^{-} \text {. }
$$

Let $x^{*}$ be an arbitrary element of $\left.(A \cap B)^{-}=(\overline{\operatorname{co}(A \cap B})\right)^{-}$. Then, by (5.3), we obtain

$$
x^{*}(x) \leqq 0 \quad \text { for all } x \in \overline{\operatorname{co}(A)} \cap \overline{\operatorname{co}(B)} .
$$

It is valid, on the basis of Lemma 5.2 , that

$$
x^{*} \in \overline{A^{-}+B^{-}} \text {. }
$$

"only if" part: It is easily seen that

$$
\overline{\operatorname{co}(A \cap B)} \subset \overline{\operatorname{co}(A)} \cap \overline{\operatorname{co}(B)} .
$$

We shall prove by contradiction. If there is a vector $y_{0} \in X$ such that

$$
y_{0} \in \overline{\operatorname{co}(A)} \cap \overline{\operatorname{co}(B)} \text { and } y_{0} \notin \overline{\operatorname{co}(A \cap B)} \text {, }
$$

then, on the basis of the separation theorem in a locally convex linear topological space (see [2], p. 417, Theorem 10), there exist a real number $\alpha$, a positive number $\varepsilon$, and a non-zero continuous linear functional $f \in X^{*}$ such that

$$
\left.f(x) \leqq \alpha<\alpha+\varepsilon \leqq f\left(y_{0}\right) \quad \text { for all } x \in \overline{\operatorname{co}(A \cap B}\right),
$$


or

$$
f(x) \leqq 0<f\left(y_{0}\right) \quad \text { for all } \quad x \in \overline{\operatorname{co}(A \cap B)},
$$

since $\overline{\operatorname{co}(A \cap B)}$ is a cone. Then, we have, by (5.2),

$$
f \in \overline{\operatorname{co}(\overline{A \cap B})^{-}}=(A \cap B)^{-}=\overline{A^{-}+B^{-}} .
$$

It is then true that there exists a generalized sequence (see [2], p. 26, Definition 1) $\left\{a_{\alpha}^{*}+b_{\alpha}^{*}\right\}, a_{\alpha}^{*} \in A^{-}, b_{\alpha}^{*} \in B^{-}$, which converges to $f$. This is equivalent to the fact that

$$
\lim _{\alpha}\left(a_{\alpha}^{*}+b_{\alpha}^{*}\right)(x)=f(x) \quad \text { for all } x \in X .
$$

Since $y_{0} \in \overline{\operatorname{co}(A)}$ and $y_{0} \in \overline{\operatorname{co}(B)}$, we have

and hence

$$
\left(a_{\alpha}^{*}+b_{\alpha}^{*}\right)\left(y_{0}\right)=a_{\alpha}^{*}\left(y_{0}\right)+b_{\alpha}^{*}\left(y_{0}\right) \leqq 0+0=0,
$$

$$
f\left(y_{0}\right)=\lim _{\alpha}\left(a_{\alpha}^{*}+b_{\alpha}^{*}\right)\left(y_{0}\right) \leqq 0 .
$$

This contradicts to (5.4). This completes the proof of Proposition 5.1.

EXAMPLE 1. Let us discuss the hypothesis under which the necessary conditions are obtained in Varaiya [8] and Guignard [3]. Let us consider the following example:

$$
\begin{aligned}
& X=R^{2}, \quad Y=R^{1}, \\
& A=\left\{\left(x_{1}, x_{2}\right) \in R^{2} \mid-1 \leqq x_{1} \leqq 1,0 \leqq x_{2} \leqq 1\right\}, \\
& f(x)=f\left(x_{1}, x_{2}\right)=x_{2}, \\
& g(x)=g\left(x_{1}, x_{2}\right)=x_{1}^{2}-x_{2}^{2}, \\
& B=\left\{r \in R^{1} \mid r \leqq 0\right\}
\end{aligned}
$$

It is clear that the optimal solution $\bar{x}$ to this problem is the origin. Note that

$$
\overline{\operatorname{co}(\operatorname{LC}(A \cap H, 0)}) \overline{(\operatorname{co}(\operatorname{LC}(A, 0))} \cap \overline{\operatorname{co}(\operatorname{LC}(H, 0))},
$$

Where $H=g^{-1}(B)$, and hence, on the basis of Proposition 5.1, we have

$$
\mathrm{LC}(A \cap H, 0)^{-} \supseteq \mathrm{LC}(A, 0)^{-}+\mathrm{LC}(H, 0)^{-} \text {. }
$$

That is, this example does not satisfy the hypothesis stated in Varaiya [8] and Guignard [3]. We can, however, treat this example since $A$ is convex, and since $B$ has a non-empty interior.

EXAMPLE 2. Let

$$
\begin{aligned}
& X=Y=R^{2} \\
& A=\left\{\left(x_{1}, x_{2}\right) \in R^{2} \mid-1 \leqq x_{1} \leqq 1,0 \leqq x_{2} \leqq 1\right\} \\
& \quad \cup\left\{\left(x_{1}, x_{2}\right) \in R^{2} \mid 0 \leqq x_{1} \leqq 1,-1 \leqq x_{2} \leqq 0\right\} \\
& B=\left\{\left(y_{1}, y_{2}\right) \in R^{2} \mid y_{1} \leqq 0, y_{2} \leqq 0\right\} \\
& g_{1}\left(x_{2}, x_{2}\right)=x_{2}-2 x_{1} \\
& g_{2}\left(x_{1}, x_{2}\right)=\frac{1}{2} x_{1}-x_{2}
\end{aligned}
$$




$$
f\left(x_{1}, x_{2}\right)=x_{2} .
$$

It is then clear that the optimal solution $\bar{x}$ is the origin. In this case, $A$ is locally relatively convex with respect to $H=g^{-1}(B)$ at 0 , though $A$ is not locally convex at 0 . Note that

$$
\mathrm{P}(A, 0)=X=R^{2},
$$

on the other hand,

$$
\mathrm{LC}(A, 0) \subsetneq X .
$$

Hence, every convex set $K$ satisfying

$$
0 \in K \subset \mathrm{LC}(A, 0),
$$

is strictly contained in $\mathrm{P}(A, 0)=X$. Consequently, our necessary conditions are different from the necessary conditions in Neustadt [5] or Nagahisa \& Sakawa [4].

EXAMPLE 3. In the last example, consider the following one. We shall show through this example that the hypotheses in Theorem 3.3 are not omitted to obtain the necessary conditions. Let

$$
\begin{aligned}
& X=Y=R^{2}, \\
& A=\left\{\left(x_{1}, x_{2}\right) \in R^{2} \mid x_{2} \leqq 0\right\} \\
& \quad \cup\left\{\left(x_{1}, x_{2}\right) \in R^{2} \mid x_{2} \geqq 0, x_{1} \leqq x_{2}^{2}\right\}, \\
& g_{1}\left(x_{1}, x_{2}\right)=-2 x_{1}-x_{2}, \\
& g_{2}\left(x_{1}, x_{2}\right)=2 x_{1}+x_{2}-2, \\
& B=\left\{\left(y_{1}, y_{2}\right) \in R^{2} \mid y_{1} \leqq 0, y_{2} \leqq 0\right\}, \\
& f\left(x_{1}, x_{2}\right)=x_{1}+x_{2} .
\end{aligned}
$$

It is then immediate that the optimal solution $\bar{x}$ is the origin. In this case, $A$ is not locally convex at 0 , nor locally relatively convex with respect to $H$ at 0 . Furthermore, note that $\mathrm{P}(A, 0)=X=R^{2}$.

For this example, we cannot obtain the necessary conditions in Theorem 3.3. Indeed, if we assume that the above necessary conditions hold, then, there are a real number $\eta$ and a vector $y^{*}=\left(y_{1}^{*}, y_{2}^{*}\right) \in R^{2}$, not both zero, such that

$$
\begin{gathered}
\eta f_{x}(z)+y_{1}^{*} g_{1 x}(z)+y_{2}^{*} g_{2 x}(z) \geqq 0 \quad \text { for all } z \in R^{2}, \\
y_{1}^{*} g_{1}(0)+y_{2}^{*} g_{2}(0)=0, \\
\eta \geqq 0, \\
y^{*}(y) \leqq 0 \quad \text { for all } y \in B .
\end{gathered}
$$

It is easily seen that

$$
\eta=y_{1}^{*}=y_{2}^{*}=0,
$$

which is a contradiction.

REMARK. This example also shows that there is a set $A(\ni 0)$ satisfying

$$
\overline{\mathrm{co}(\mathrm{LC}(A, 0))} \varsubsetneqq \mathrm{P}(A, 0) \text {. }
$$


Acknowledgement. The author is indebted to Dr. Tosio Kitagawa for his valuable comments to the original version of this paper.

\section{References}

[1] N. Bourbaki, Espaces Vectoriels Topologiques, Hermann, Paris, 1966.

[2] N. Dunford and J. T. Schwartz, Linear Operators, Part I, Interscience, New York, 1964.

[3] M. Guignard, Generalized Kuhn-Tucker conditions for mathematical programming problems in a Banach space, SIAM J. Control, 7 (1969), 232-241.

[4] Y. NAGAHISA and Y. SAKAwA, Nonlinear programming in Banach spaces, J. Optimization Theory and Applications, 4 (1969), 182-190.

[5] L.W. Neustadt, A general theory of extremals, J. Computer and System Sciences, 3 (1969), 57-92.

[6] L.W. Neustadt, An abstract variational theory with applications to a broad class of optimizatisn problems. I. General theory, J. SIAM Control, 4 (1966), pp. 505-527.

[7] R. T. Rockafellar, Convex analysis, Princeton University Press, 1970.

[8] P. VARAIYA, Nonlinear programming in Banach space, SIAM J. Appl. Math., 15 (1967), 284-293.

[9] S. ZlOBEC, Asymptotic Kuhn-Tucker conditions for mathematical programming problems in a Banach space, SIAM J. Control, 8 (1970), 505-512. 